T. Kawasaki

Nagoya Math. J.

Vol. 84 (1981), 135-157

\title{
THE INDEX OF ELLIPTIC OPERATORS OVER $V$-MANIFOLDS
}

\author{
TETSURO KAWASAKI
}

\section{Introduction}

Let $M$ be a compact smooth manifold and let $G$ be a finite group acting smoothly on $M$. Let $E$ and $F$ be smooth $G$-equivariant complex vector bundles over $M$ and let $P: \mathscr{C}^{\infty}(M ; E) \rightarrow \mathscr{C}^{\infty}(M ; F)$ be a $G$-invariant elliptic pseudo-differential operator. Then the kernel and the cokernel of the operator $P$ are finite-dimensional representations of $G$. The difference of the characters of these representations is an element of the representation ring $R(G)$ of $G$ and is called the $G$-index of the operator $P$.

$$
\text { ind } P=\operatorname{char}[\text { kernel } P]-\operatorname{char}[\operatorname{cokernel} P] \text {. }
$$

It is well-known that the $G$-index ind $P \in R(G)$ depends only on the homotopy class of the elliptic operator and, as Atiyah and Singer showed in [2], ind $P$ is determined by the stable equivalence class $[\sigma(P)] \in K_{G}(\tau M)$ of the principal symbol $\sigma(P)$ viewed as the difference bundle over the tangent bundle $\tau M$. The Atiyah-Singer index theorem asserts that the value (ind $P)(g)$ is expressed by the evaluation of a certain characteristic class over the tangent bundle $\tau\left(M^{g}\right)$ of the fixed point set $M^{g}$.

$$
\text { (ind } P)(g)=(-1)^{\operatorname{dim} M^{g}}\left\langle\operatorname{ch}^{g}[\sigma(P)] \mathscr{I}^{g}(M),\left[\tau\left(M^{g}\right)\right]\right\rangle \text {. }
$$

Here $\operatorname{ch}^{g}[\sigma(P)]$ is a class in the compactly supported cohomology group $H_{c}^{*}\left(\tau\left(M^{g}\right) ; C\right)$ expressed in the characteristic classes of the complex eigenvector bundles by the action of $g$ on the stable vector bundle $\left[\left.\sigma(P)\right|_{\tau\left(M M^{g}\right)}\right]$. $\mathscr{I}^{g}(M)$ is a class in $H^{*}\left(M^{g} ; C\right)$ expressed in the characteristic classes of the real and complex eigenvector bundles by the action of $g$ on the real vector bundle $\left.\tau M\right|_{M} g$. We call these classes over the fixed point set as the residual characteristic classes.

Next we consider the index of the operator $P^{G}: \mathscr{C}^{\infty}(M ; E)^{G} \rightarrow \mathscr{C}^{\infty}(M ; F)^{G}$

Received December 17, 1979. 
between $G$-invariant sections. By the orthonormality of irreducible characters, we have:

$$
\text { ind } \begin{aligned}
P^{G} & =\operatorname{dim}\left[\text { kernel } P^{G}\right]-\operatorname{dim}\left[\text { cokernel } P^{G}\right] \\
& =\frac{1}{|G|} \sum_{g \in G}(\text { ind } P)(g) \\
& =\frac{1}{|G|} \sum_{g \in G}(-1)^{\operatorname{dim} M^{g}}\left\langle\operatorname{ch}^{g}[\sigma(P)] \mathscr{I}^{g}(M),\left[\tau\left(M^{g}\right)\right]\right\rangle .
\end{aligned}
$$

The operator $P^{a}$ can be viewed as an operator over the orbit space $G \backslash M$ in the following sense. The invariant section $s: M \rightarrow E$ is determined uniquely by the induced section $\bar{s}: G \backslash M-G \backslash E$ over the orbit space. So we may consider the invariant sections $\mathscr{C}^{\infty}(M ; E)^{a}$ as the sections over the orbit space $X=G \backslash M$. The operator $P^{G}$ operates on these sections and its index ind $P^{G}$ depends only on the $G$-equivariant homotopy class of the principal symbol $[\sigma(P)]$, which is considered to be a section over the orbit space $G \backslash \tau M$. Thus we consider $P^{G}$ as an operator over $X=G \backslash M$.

We remark that the evaluation in (3) admits a purely local expression over $X$. Choose $G$-invariant metrics and connections on manifolds $M$ and $M^{g}$, on bundles $\tau M, \tau\left(M^{g}\right)$ and $\nu\left(M^{g}\right)$ (the normal bundle of $M^{g}$ in $M$ ) and on a stable bundle $\sigma(P)$. Then the evaluations of residual characteristic classes are given by the integrations of the corresponding characteristic forms. For each $x \in M$, we choose a small neighbourhood $U_{x}$ so that the isotropy subgroup $G_{x}$ acts on $U_{x}$ and, for $g \in G, U_{x} \cap g U_{x} \neq \emptyset$ implies $g \in G_{x}$. Then the orbit space $G_{x} \mid U_{x}$ is naturally identified with an open subset in $X$. A family $\left\{G_{x} \backslash U_{x}\right\}_{x \in M}$ defines an open covering of $X$. Choose a partition of unity $1=\sum \phi_{x}$ subordinate to this coverinig. Then we can rewrite (3) in the following form

(4) ind $P^{a}=\sum_{x \in M} \frac{1}{\left|G_{x}\right|} \sum_{g \in G_{x}}(-1)^{\operatorname{dim} U_{x}^{g}} \int_{\tau\left(U_{x}^{g}\right)} \phi_{x} \operatorname{ch}^{g}\left[\left.\sigma(P)\right|_{U_{x}}\right] \mathscr{I}^{g}\left(U_{x}\right)$.

The orbit space $G \backslash M$ is a typical example of $V$-manifold, and the above formula (4) can be given an interpretation which still makes sense for general $V$-manifolds.

The purpose of the present paper is to give an index theorem for elliptic operators over $V$-manifolds which generalize the formula (4).

Let $X$ be a compact $V$-manifold. (For the precise definitions of $V$ manifolds and $V$-bundles, see Kawasaki [6]). For each $x \in X$, there is a neighbourhood $U_{x}$ and an identification $U_{x}=G_{x} \mid \tilde{U}_{x}$, where $\tilde{U}_{x}$ is a 
neighbourhood of the origin in an effective real representation space of a finite group $G_{x}$. For each $y \in U_{x}$, choose small $U_{y}$ so that $U_{y} \subset U_{x}$, then there is an open embedding $\phi: \tilde{U}_{y} \rightarrow \tilde{U}_{x}$ that covers the inclusion $U_{y}$ $\cdot \subset U_{x}$. The choice of such $\phi$ is unique up to the action of $G_{x}$ on $\tilde{U}_{x}$. Each $\phi$ determines an injective group homomorphism $\lambda_{\phi}: G_{y} \rightarrow G_{x}$ that makes $\phi$ be $\lambda_{\phi}$-equivariant.

To express our theorem in cohomological terms, we have to assign to each $V$-manifold $X$ a certain global geometric object over which the residual characteristic classes should be evaluated. If we look at (4), such an object must be a collection of all $\tilde{U}_{x}^{g}$ 's. Each $\tilde{U}_{x}^{g}$ admits the action of the centralizer $Z_{G_{x}}(g)$ of $g$ in $G_{x}$. If $g$ and $g^{\prime}$ are conjugate in $G_{x}$, then $U_{x}^{g}$ and $U_{x}^{g^{\prime}}$ are diffeomorphic by the action of some element $h$ in $G_{x}\left(g^{\prime}=h g h^{-1}\right)$. So we consider one element $g$ for each conjugacy class (g) in $G_{x}$. For each point $x \in X$, let $(1),\left(h_{x}^{1}\right), \cdots,\left(h_{x}^{\rho x}\right)$ be all the conjugacy classes in $G_{x}$. Then we have a natural bijection

$$
\begin{aligned}
& \left\{\left(y,\left(h_{y}^{j}\right)\right) \mid y \in U_{x}, j=1,2, \cdots, \rho_{y}\right\} \\
& \cong \prod_{i=1}^{\rho_{x}} Z_{G_{x}}\left(h_{x}^{i}\right) \backslash \tilde{U}_{x}^{h_{x}^{i}} .
\end{aligned}
$$

So we define globally:

$$
\Sigma X=\left\{\left(x,\left(h_{x}^{i}\right)\right) \mid x \in X, G_{x} \neq\{1\}, i=1,2, \cdots, \rho_{x}\right\}
$$

Then $\Sigma X$ has a natural $V$-manifold structure whose local coordinate coverings are $\tilde{U}_{x}^{h} \rightarrow Z_{G_{x}}(h) \backslash \tilde{U}_{x}^{h}(h \neq 1)$. The action of $Z_{G_{x}}(h)$ on $\tilde{U}_{x}^{h}$ is not effective. The order of the trivially acting subgroup is called the multiplicity of $\Sigma X$ in $X$ at $(x,(h))$. In general, $\Sigma X$ has many connected components of varying dimensions. Let $\Sigma_{1}, \Sigma_{2}, \cdots, \Sigma_{c}$ be the connected components of $\Sigma X$. Since the multiplicity is locally constant on $\Sigma X$, we may assign the multiplicity $m_{i}$ to each connected component $\Sigma_{i}$.

On each local coordinate $\tilde{U}_{x}^{h}$ over $\Sigma X$, we have the normal bundle $\nu\left(\tilde{U}_{x}^{h}\right)$ in $\tilde{U}_{x}$ and the tangent bundle $\tau\left(\tilde{U}_{x}^{h}\right)$. On the normal bundle $\nu\left(\tilde{U}_{x}^{h}\right)$, we have the action of $h$. Then we have the eigenspace decomposition of $\nu\left(\tilde{U}_{x}^{h}\right)$

$$
\begin{aligned}
& \nu\left(\tilde{U}_{x}^{h}\right)=\underset{0<\theta \leqq \pi}{\bigoplus} \nu_{h}^{\theta}, \\
& \begin{cases}h v=e^{i \theta} v & \text { if } v \in \nu_{h}^{\theta}(0<\theta<\pi), \\
h v=-v & \text { if } v \in \nu_{h}^{\pi} .\end{cases}
\end{aligned}
$$


The collection of these $Z_{\sigma_{x}}(h)$-equivariant bundles $\nu_{h}^{\theta}(0<\theta \leqq \pi)$ and $\tau\left(\tilde{U}_{x}^{h}\right)$ form a real or complex vector $V$-bundles over $\Sigma X$. By choosing invariant connections, we have a collection of residual characteristic forms

$$
\mathscr{I}^{h}\left(\tilde{U}_{x}\right) \in \Omega^{*}\left(\tilde{U}_{x}^{h}\right) \otimes_{R} C .
$$

These forms define characteristic classes

$$
\mathscr{I}^{\Sigma}(X) \in H^{*}(\Sigma X ; C), \quad \text { and } \quad \mathscr{I}(X) \in H^{*}(X ; \boldsymbol{Q}) \quad(h=1) .
$$

By a $V$-bundle $E$ over a $V$-manifold $X$, we mean a family $\left\{\left(G_{x}^{E}, \tilde{E}_{x} \rightarrow\right.\right.$ $\left.\left.\tilde{U}_{x}\right)\right\}$ of equivariant fibre bundles with surjective homomorphisms $G_{x}^{E} \rightarrow G_{x}$ and their attaching bundle maps $\{\Phi\}: \tilde{E}_{v} \rightarrow \tilde{E}_{x}$ for each inclusive pair $U_{y}$ $\subset U_{x}$. We call $V$-bundle $E$ to be proper if, for each $x \in X, G_{x}^{E}=G_{x}$. The attaching bundle maps $\{\Phi\}$ define a unique induced open embedding $\bar{\Phi}: G_{x}^{E} \backslash \tilde{E}_{y} \rightarrow G_{x}^{E} \backslash \tilde{E}_{x}$ of the orbit spaces of total spaces. These induced maps define the total space $E=\cup\left(G_{x}^{E} \backslash \tilde{E}_{x}\right)$ and the projection $E \rightarrow X . \quad E$ itself admit a structure of $V$-manifold.

Let $E \rightarrow X$ be a proper $V$-bundle. A section $s: X \rightarrow E$ is called a $C^{\infty}$ $V$-section if, for each $U_{x}, s\left|U_{x}: U_{x} \rightarrow E_{x}=G_{x}\right| \tilde{E}_{x}$ is covered by a $G_{x}$ invariant $C^{\infty}$ section $\tilde{s}_{x}: \tilde{U}_{x} \rightarrow \tilde{E}_{x}$. For a vector $V$-bundle $E$, we denote the set of all $C^{\infty} V$-sections by $\mathscr{C}_{V}^{\infty}(X ; E)$, which forms a vector space. On a vector $V$-bundle $E$, we can always construct a invariant linear connection, that is, a family of invariant connections on $\left(G_{x}^{E}, \tilde{E}_{x} \rightarrow \tilde{U}_{x}\right)$ which are compatible with attaching bundle maps. Then the characteristic forms define a $C^{\infty} V$-section of the exterior power of the cotangent vector $V$ bundle, which represent a cohomology class on $X$.

Let $E$ and $F$ be proper complex vector $V$-bundles over $X$. A linear map $P: \mathscr{C}_{V}^{\infty}(X ; E) \rightarrow \mathscr{C}_{V}^{\infty}(X ; F)$ is called a (pseudo-) differential operator if locally it is covered by invariant (pseudo-) differential operators

$$
\tilde{P}_{x}: \mathscr{C}_{c}^{\infty}\left(\tilde{U}_{x} ; \tilde{E}_{x}\right)-\mathscr{C}^{\infty}\left(\tilde{U}_{x} ; \tilde{F}_{x}\right)
$$

(modulo smoothing operators), which are compatible with attaching maps. We call $P$ to be elliptic if each $\tilde{P}_{x}$ is elliptic. For an elliptic pseudodifferential operator $P: \mathscr{C}_{V}^{\infty}(X ; E) \rightarrow \mathscr{C}_{V}^{\infty}(X ; F)$, we have the $V$-index defined by:

$$
\operatorname{ind}_{V} P=\operatorname{dim}[\operatorname{kernel} P]-\operatorname{dim}[\text { cokernel } P] \text {. }
$$

This index generalize ind $P^{a}$ in (3) and (4). 
Like $G$-equivariant case, the $V$-index depends only on the homotopy class of elliptic operators. The principal symbol $\sigma(P)$ of the operator $P$ is a well-defined $C^{\infty} V$-section of the $V$-bundle $\operatorname{Hom}(E, F)$ over the total space $\tau_{V}^{*} X$ of the cotangent vector $V$-bundle. For $P$ elliptic, the principal symbol $\sigma(P)$ defines a compactly supported difference $V$-bundle and the index $\operatorname{ind}_{V} P$ is determined by its stable equivalence class $[\sigma(P)]$. The stable equivalence classes of compactly supported proper difference vector $V$-bundles over $\tau_{V}^{*} X \cong \tau_{V} X$ form a group $K_{V}\left(\tau_{V}^{*} X\right) \cong K_{V}\left(\tau_{V} X\right) . \quad\left(\tau_{V} X\right.$ denotes the total space of the tangent vector $V$-bundle). Then $V$-index defines a homomorphism

$$
\operatorname{ind}_{V}: K_{V}\left(\tau_{V} X\right) \longrightarrow Z
$$

An element $u \in K_{V}\left(\tau_{V} X\right)$ is represented by proper complex vector $V$ bundles $E$ and $F$ over $\tau_{V} X$ and an isomorphism $\sigma: E \rightarrow F$ over $\tau_{V} X-X$. Then, choosing a suitable invariant connections, we have the residual Chern characters

$$
\operatorname{ch}^{h}(E)-\operatorname{ch}^{h}(F) \in \Omega^{*}\left(\tau\left(\tilde{U}_{x}^{h}\right)\right) \bigotimes_{R} C,
$$

and globally we have the classes

$$
\operatorname{ch}^{\Sigma}(u) \in H_{c}^{*}\left(\tau_{V}(\Sigma X) ; C\right) \text { and } \operatorname{ch}(u) \in H_{c}^{*}\left(\tau_{V} X ; Q\right) \quad(h=1) .
$$

In this framework, we can state our theorem

Theorem. Let $X$ be a compact $V$-manifold. Then, for $u \in K_{V}\left(\tau_{V} X\right)$, we have:

$$
\begin{aligned}
\operatorname{ind}_{V}(u)= & (-1)^{\operatorname{dim} X}\left\langle\operatorname{ch}(u) \mathscr{I}(X),\left[\tau_{V} X\right]\right\rangle \\
& +\sum_{i=1}^{c} \frac{(-1)^{\operatorname{dim} \Sigma_{i}}}{m_{i}}\left\langle\operatorname{ch}^{\Sigma}(u) \mathscr{I}^{\Sigma}(X),\left[\tau_{V} \Sigma_{i}\right]\right\rangle .
\end{aligned}
$$

As a special case of this theorem, we get the following results:

I) (Kawasaki [6]) Let $X$ be a compact oriented $V$-manifold of dimension $4 k$. As a topological space, $X$ is an oriented rational homology manifold. The signature $\operatorname{Sign}(X)$ of $X$ is defined by the signature of the non-degenerate symmetric bilinear form on the middle dimensional cohomology group $H^{2 k}(X ; \boldsymbol{Q})$ given by the cup product. Using de Rham cohomology, we can represent $\operatorname{Sign}(X)$ as the $V$-index of the signature operator $D_{+}: \Omega_{V}^{+}(X) \rightarrow \Omega_{\bar{V}}^{-}(X)$ over $V$-manifold $X$. Then we have: 


$$
\operatorname{Sign}(X)=\langle L(X),[X]\rangle+\sum_{i=1}^{c} \frac{1}{m_{i}}\left\langle L^{\Sigma}(X),\left[\Sigma_{i}\right]\right\rangle .
$$

The classes $L(X)$ and $L^{\Sigma}(X)$ are defined locally by the residual $L$-class $L^{h}\left(\tilde{U}_{x}\right)$, as we have defined $\mathscr{I}(X)$ and $\mathscr{I}^{2}(X)$.

II) (Kawasaki [7]) Let $X$ be a compact complex $V$-manifold and let $E \rightarrow X$ be a holomorphic vector $V$-bundle. Then $X$ admits a natural structure of an analytic space and the local holomorphic $V$-sections of $E$ define a coherent analytic sheaf $\mathcal{O}_{V}(E)$ over $X$. The arithmetic genus $\chi(X ; E)$ is defined by:

$$
\chi(X ; E)=\sum_{i=1}^{\operatorname{dim} X}(-1)^{i} \operatorname{dim}_{C} H^{i}\left(X ; \mathcal{O}_{V}(E)\right) .
$$

Then $\chi(X ; E)$ is represented by the $V$-index of the Dolbeault complex over the $V$-manifold $X$ with coefficients in $E$. We can apply our theorem and we have:

$$
\chi(X ; E)=\langle\mathscr{T}(X ; E),[X]\rangle+\sum_{i=1}^{c} \frac{1}{m_{i}}\left\langle\mathscr{T}^{\Sigma}(X ; E),\left[\Sigma_{i}\right]\right\rangle .
$$

The classes $\mathscr{T}(X ; E)$ and $\mathscr{T}^{\Sigma}(X ; E)$ are defined locally by the residual Todd class with coefficients in $E$.

The proof that we adopt here is completely different from those in the above two reports [6] and [7]. As we have remarked in [6], every $V$ manifold $X$ is presented as the orbit space of a smooth $G$-manifold $\tilde{X}$ with only finite isotropy subgroups and with the trivial principal orbit type. We may choose such $(G, \tilde{X})$ with $G$ compact and connected. Let $P$ be an elliptic operator over $X$. Then we can lift the principal symbol $\sigma(P)$ considered as a difference $V$-bundle over $\tau_{V} X$ to a $G$-equivariant difference bundle over $\tau_{G} \tilde{X}$, the space of tangent vectors orthogonal to the orbits of $G$. The lifted symbol determines up to homotopy a transversally elliptic operator $\tilde{P}$ over $\tilde{X}$ relative to $G$. Then the $V$-index $\operatorname{ind}_{V} P$ is equal to the evaluation $\left(\operatorname{ind}^{\sigma} \tilde{P}\right)\left(1_{G}\right)$ of the distributional index $\operatorname{ind}^{\sigma} \tilde{P}$ by the unit function over $G$.

For the distributional index of transversally elliptic operators, we refer to Atiyah [1]. We use two main results of [1]. One result is an expression of $\operatorname{ind}^{T} P$, for a transversally elliptic operator $P$ over a manifold $M$ relative to a toral action with only finite isotropy subgroups. The value $\left(\operatorname{ind}^{T} P\right)\left(1_{T}\right)$ is written by the evaluation of the equivariant residual characteristic classes over the orbit spaces $T \backslash \tau_{T} M^{h}\left(h \in T, M^{h} \neq \phi\right)$ (including 
$h=1)$. By a direct translation, this formula gives the formula (7) in our theorem, when the $V$-manifold $X$ has the form $X=T \backslash M$. Another result is a reduction formula $\left(\operatorname{ind}^{G} P\right)\left(1_{G}\right)=\left(\operatorname{ind}^{T}([\bar{\partial}] \otimes P)\right)\left(1_{T}\right)$, for a compact connected Lie group $G$, where $T$ is a maximal torus of $G$ and [ō] denotes the Dolbeault complex over the flag manifold $G / T$.

Combining these two results, we get an expression of the $V$-index using the evaluation of characteristic classes over an auxiliary $V$-manifold $T \backslash \tilde{X}$ and its singularities. This new $V$-manifold $T \backslash \tilde{X}$ is a fibration (with singularities) over $X$ with generic fibre $G / T$. We apply the Gysin homomorphism (the integration over the fibre) to these characteristic classes. Then we get classes over the $V$-manifold $X$ and its singularities. To deduce (7), we need a formula on the equivariant residual Todd classes over the flag manifold $G / T$. This formula is a generalization of the following result in Borel-Hirzebruch [5].

Let $G$ be a compact connected Lie group and let $T$ be a maximal torus of $G$. We fix a $G$-invariant complex structure on the flag manifold $G / T$. Consider the fibration $\pi: B T \rightarrow B G$ of classifying spaces with fibre $G / T$. Its bundle along the fibre is a complex vector bundle over $B T$. We denote by $\mathscr{T}_{G}(G / T)$ the Todd class of this bundle. (This class is the $G$-equivariant Todd class of the complex $G$-manifold $G / T)$. Then Borel and Hirzebruch proved the following:

TheOREM (Borel-Hirzebruch [5]). Let $\pi_{1}: H^{* *}(B T ; \boldsymbol{R}) \rightarrow H^{* *}(B G ; \boldsymbol{R})$ be the Gysin homomorphism (the integration over the fibre). Then we have:

$$
\pi_{!} \mathscr{T}_{G}(G / T)=1 \in H^{* *}(B G ; R)=H_{G}^{* *}(p t ; R),
$$

where $H_{G}^{* *}$ denotes the completed equivariant cohomology group for G-spaces.

Let $h \in T$ be an element. The action of $h$ on $G / T$ is holomorphic. So the fixed point set $(G / T)^{h}$ is a complex submanifold (non-connected) with the holomorphic action of the centralizer $Z_{G}(h)$. The tangent bundle $\tau_{h}$ and the normal bundle $\nu_{h}$ are the $Z_{G}(h)$-equivariant complex vector bundles. Let $\nu_{h}=\oplus \nu_{h}^{\theta}$ be the eigenspace decomposition by the action of $h$. Then we define the equivariant residual Todd class by:

$$
\begin{aligned}
\mathscr{T}_{G}^{h}(G / T)= & \mathscr{T}_{Z_{G}(h)} \prod_{0<\theta<2 \pi} \mathscr{T}_{Z_{G}(h)}^{\theta}\left(\nu_{h}^{\theta}\right) \\
= & \mathscr{T}\left(E Z_{G}(h) \times_{Z_{G(h)}} \tau_{h}\right) \prod_{0<\theta<2 \pi} \mathscr{T}^{\theta}\left(E Z_{G}(h) \times_{Z_{G(h)}} \nu_{h}^{\theta}\right) \\
& \in H_{Z_{G}(h)}^{* *}\left((G / T)^{h} ; C\right)=H^{* *}\left(E Z_{G}(h) \times_{Z_{G(h)}}(G / T)^{h} ; C\right) .
\end{aligned}
$$


The base space $E Z_{G}(h) \times_{z_{G}(h)}(G / T)^{h}$ is a fibration over $B Z_{G}(h)$ with the fibre $(G / T)^{h}$. Then we have the Gysin homomorphism $\pi_{!}: H_{Z_{g}(h)}^{* *}\left((G / T)^{h} ; C\right)$ $\rightarrow H_{Z_{(}(h)}^{* *}(p t ; C)=H^{* *}\left(B Z_{G}(h ; C)\right.$.

Theorem. The Gysin homomorphism of the equivariant residual Todd class is given by:

$$
\pi_{!} \mathscr{T}_{G}^{h}(G / T)=1 \in H^{* *}\left(B Z_{G}(h) ; C\right)=H_{Z_{G}(h)}^{* *}(p t ; C) .
$$

If we put $h=1$, we recover (8). The proof of this formula is straightforward. The same technique as in Borel-Hirzebruch [4] is applicable. We can express $\pi_{1} \mathscr{T}_{G}^{h}(G / T) \in H^{* *}\left(B Z_{G}(h) ; C\right) \subset H^{* *}(B T ; C)$ in the power series in the roots of the Lie group $G$. Then we deduce our formula from the Weyl's relation on the roots of $G$.

\section{§1. Distributional index and $V$-index}

In this section we summarize the results in Atiyah [1] that we need and we shall show the relation between the distributional index of transversally elliptic operators and the $V$-index of elliptic operators over $V$ manifolds.

Let $G$ be a compact Lie group and let $M$ be a compact smooth $G$ manifold without boundary. We choose a $G$-invariant Riemannian metric on $M$ and we identify the cotangent bundle $\tau^{*} M$ and the tangent bundle $\tau M$. We define a subset $\tau_{G} M$ in $\tau M$ as the set of all the tangent vectors that are orthogonal to the orbits of $G$.

Let $E$ and $F$ be $G$-equivariant smooth complex vector bundles over $M$ and let $P: \mathscr{C}^{\infty}(M ; E) \rightarrow \mathscr{C}^{\infty}(M ; F)$ be a $G$-invariant pseudo-differential operator of order $m$. By choosing invariant metrics and invariant connections on $E$ and $F$, we have the space of Sobolev sections $\mathscr{H}^{s}(M ; E)$ and $\mathscr{H}^{s}(M ; F)(s \in \boldsymbol{R})$. Then the operator $P$ extends uniquely to a bounded operator $P: \mathscr{H}^{s}(M ; E) \rightarrow \mathscr{H}^{s-m}(M ; F)$. Also we have the adjoint operator $P^{*}: \mathscr{H}^{s}(M ; F) \rightarrow \mathscr{H}^{s-m}(M ; E)$. The null spaces $\mathscr{N}^{s}(P)$ and $\mathscr{N}^{s}\left(P^{*}\right)$ are closed subspaces and admit the structure of Hilbert spaces. We may consider $\mathscr{N}^{s}(P)$ and $\mathscr{N}^{s}\left(P^{*}\right)$ as unitary representations of $G$. We denote by $\hat{G}$ the set of all equivalence classes of irreducible representations of $G$. For $\alpha \in \hat{G}$, we denote the $\alpha$-components by $\mathscr{N}_{\alpha}^{s}(P)$ and $\mathscr{N}_{\alpha}^{s}\left(P^{*}\right)$.

We call a $G$-invariant pseudo-differential operator $P: \mathscr{C}^{\infty}(M ; E) \rightarrow$ $\mathscr{C}^{\infty}(M ; F)$ to be transversally elliptic relative to $G$ if the principal symbol $\sigma(P)$ is invertible over $\tau_{G} M-M$. Then we have: 
Theorem (Atiyah [1]). Let $P: \mathscr{C}^{\infty}(M ; E) \rightarrow \mathscr{C}^{\infty}(M ; F)$ be a transversally elliptic operator. Then for each $\alpha \in \hat{G}, \mathscr{N}_{\alpha}^{s}(P)$ is finite dimensional and does not depend on s. Furthermore the formal sum

$$
\operatorname{char} \mathscr{N}(P)=\sum_{\alpha \in \hat{Q}} \operatorname{char} \mathscr{N}_{\alpha}^{s}(P)
$$

converges in $\mathscr{H}^{-n-s}(G)(n=\operatorname{dim} M)$ for any $\varepsilon>0$.

Now we can define the distributional index:

Definition. Let $P: \mathscr{C}^{\infty}(M ; E) \rightarrow \mathscr{C}^{\infty}(M ; F)$ be a transversally elliptic operator relative to $G$. Then the distributional index $\operatorname{ind}^{\sigma}(P)$ is defined by:

$$
\operatorname{ind}^{G}(P)=\operatorname{char} \mathscr{N}(P)-\operatorname{char} \mathscr{N}\left(P^{*}\right) \in \mathscr{D}^{\prime}(G)^{\mathrm{inv}}
$$

Here we denote by $\mathscr{D}^{\prime}(G)^{\text {inv }}$ the distributions on $G$ invariant under the inner automorphisms of $G$.

The distributional index has the following properties:

Theorem (Atiyah [1]). The distributional index of a transversally elliptic operator $P$ depends only on the homotopy class of the restriction of the principal symbol $\sigma(P)$ to $\tau_{G} M-M$

$$
\left.\sigma(P)\right|_{r_{G M-M}} \in \text { Iso }\left.\left(\pi^{*} E, \pi^{*} F\right)\right|_{\tau_{G M-M}} .
$$

Corollary. The distributional index defines a $R(G)$-module homomorphism

$$
\operatorname{ind}^{G}: K_{G}\left(\tau_{G} M\right) \longrightarrow \mathscr{D}^{\prime}(G)^{\text {inv }} .
$$

For each $\alpha \in \hat{G}$, the transversally elliptic operator $P$ defines a $G$ invariant Fredholm operator

$$
P_{\alpha}: \mathscr{H}_{\alpha}^{s}(M ; E) \longrightarrow \mathscr{H}_{\alpha}^{s-m}(M ; F) .
$$

So we may consider $\operatorname{ind}^{\theta}(P)=\sum_{\alpha}$ ind $\left(P_{\alpha}\right)$. Then by the orthonormality of irreducible characters, we have:

$$
\left(\operatorname{ind}^{G} P\right)\left(1_{G}\right)=\text { index }\left[P^{a}: \mathscr{C}^{\infty}(M ; E)^{a} \longrightarrow \mathscr{C}^{\infty}(M ; F)^{G}\right] \text {. }
$$

Now we assume that the action of $G$ on $M$ is of trivial principal orbit type and with only finite isotropy subgroups. Then, by definition, the above number is the $V$-index of the elliptic operator $P^{G}: \mathscr{C}_{V}^{\infty}(G \backslash M ; G \backslash E)$ $\rightarrow \mathscr{C}_{V}^{\infty}(G \backslash M ; G \backslash F)$ over the $V$-manifold $G \backslash M$. Each $G$-equivariant bundle 
$E \rightarrow M$ defines a proper $V$-bundle $G \backslash E \rightarrow G \backslash M$, and vice versa. The $V$ manifold $G \backslash \tau_{G} M$ is exactly the total space $\tau_{V}(G \backslash M)$ of the tangent $V$-bundle. Then we have the canonical isomorphism $K_{G}\left(\tau_{G} M\right) \cong K_{V}\left(\tau_{V}(G \backslash M)\right)$ and the following commutative diagram

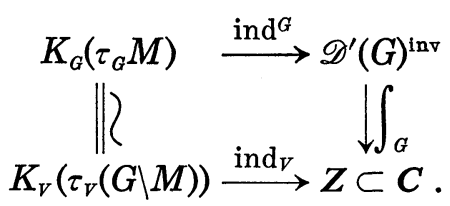

Conversely, given a $V$-manifold $X$, we choose a Riemannian metric on $X$. Then the total space $O(n)\left(\tau_{V} X\right)$ of the associated tangential orthonormal frame $V$-bundle is a smooth manifold. The right action of $O(n)$ is of trivial principal orbit type and with only finite isotropy subgroups. Its orbit space is canonically identified with the original $V$-manifold $X$. If we choose an injective homomorphism of $O(n)$ into a compact connected Lie group $G$, then the total space $\tilde{X}=O(n)\left(\tau_{V} X\right) \times_{o(n)} G$ of the associated tangential $G$-principal $V$-bundle is a smooth manifold with a right $G$ action and its orbit space is again a $V$-manifold $X$. So we recover the original situation and we also have an identification $K_{V}\left(\tau_{V} X\right) \cong K_{G}\left(\tau_{G} \tilde{X}\right)$. Thus we reduce the computations of $V$-index into those of distributional index.

For the computations of distributional index, we write down some of the results in Atiyah [1]. Let $G$ be a compact connected Lie group and let $T$ be its maximal torus. We choose and fix a $G$-invariant complex structure on the flag manifold $G / T$. Then we have the Dolbeault complex on $G / T$ and we consider its symbol [ $\bar{\partial}]$ as an element of $K_{G}(\tau(G / T))$. Let $M$ be a smooth $G$-manifold with only finite isotropy subgroups. We have a $G$-equivariant diffeomorphism $G \times{ }_{T} M \cong G / T \times M$ by sending $(g, x) \in$ $G \times{ }_{T} M$ to $(g T, g x)$. Then we have the equivalences of vector bundles

$$
G \times{ }_{T} \tau_{T} M \cong \tau_{G}\left(G \times{ }_{T} M\right) \cong \tau_{G}(G / T \times M) \cong \tau(G / T) \times \tau_{G} M .
$$

The first equivalence comes from the $(G \times T)$-equivariant bundle map $G$ $\times \tau_{T} M=\tau_{G \times T}(G \times M)$, where $G \times T$ acts on $G \times M$ by $(g, h)\left(g^{\prime}, x\right)=$ $\left(g g^{\prime} h^{-1}, h x\right)$. The third equivalence comes from the natural identification $\tau(G / T \times M) \cong \tau(G / T) \times \tau M$. Then we define a homomorphism $r: K_{G}\left(\tau_{G} M\right)$ $\rightarrow K_{T}\left(\tau_{T} M\right)$ by:

$$
r: K_{G}\left(\tau_{G} M\right) \stackrel{[\bar{\partial}] \times}{\longrightarrow} K_{G}\left(\tau(G / T) \times \tau_{G} M\right) \cong K_{G}\left(G \times_{T} \tau_{T} M\right) \cong K_{T}\left(\tau_{T} M\right) .
$$


By this homomorphism, we can compute ind ${ }^{G}$ through ind ${ }^{T}$.

THEOREM (Atiyah [1]). Let $M$ be a compact smooth G-manifold without boundary (with only finite isotropy subgroups)*). Then the following diagram commutes:

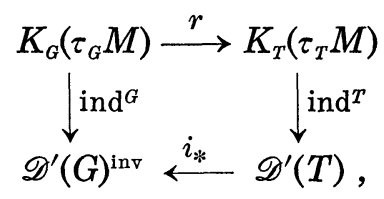

where $i_{*}: \mathscr{D}^{\prime}(T) \rightarrow \mathscr{D}^{\prime}(G)^{\mathrm{inv}}$ is the dual of the restriction $i^{*}: \mathscr{C}^{\infty}(G)^{\mathrm{inv}} \rightarrow \mathscr{C}^{\infty}(T)$.

Especially, for $u \in K_{G}\left(\tau_{G} M\right)$, we have:

$$
\left(\text { ind }^{G} u\right)\left(1_{G}\right)=\left(\operatorname{ind}^{T} r u\right)\left(1_{T}\right) \text {. }
$$

Another result that we need is the following:

Theorem (Atiyah [1]). Let $M$ be a compact smooth T-manifold without boundary, with only finite isotropy subgroups. Then for $u \in K_{T}\left(\tau_{T} M\right)$, we have:

$$
\left(\operatorname{ind}^{T} u\right)\left(1_{T}\right)=\sum_{\substack{h \in T, M^{h} \neq \phi \\ M_{i}^{h} \subset M^{h}}} \frac{(-1)^{\operatorname{dim}\left(T \backslash M_{i}^{h}\right)}}{m_{T}\left(M_{i}^{h}\right)}\left\{\operatorname{ch}_{T}^{h}(u) \mathscr{I}_{T}^{h}(M)\right\}\left[T \backslash \tau_{T} M_{i}^{h}\right],
$$

where $M_{i}^{h}$ moves over the connected components of $M^{h}$ and for each $M_{i}^{h}$, we define the multiplicity $m_{T}\left(M_{i}^{h}\right)$ by:

$$
m_{T}\left(M_{i}^{h}\right)=\text { the order of }\left\{g \in T \mid g x=x \text {, for any } x \in M_{i}^{h}\right\}^{* *)} .
$$

We review the definitions of $\operatorname{ch}_{T}^{h}(u)$ and $\mathscr{I}_{T}^{h}(u)$. Let $i_{h}: \tau_{T} M^{h} \rightarrow \tau_{T} M$ be the inclusion, then $i_{h}^{*} u \in K_{T}\left(\tau_{T} M^{h}\right)$ admits the eigenspace decomposition $i_{h}^{*} u=\oplus_{0 \leqq \theta<2 \pi} u_{h}^{\theta}$, where $u_{h}^{\theta} \in K_{T}\left(\tau_{T} M^{h}\right)$ is the stable eigenvector bundle of eigenvalue $e^{i \theta}$. Then we have an element $\operatorname{ch}_{T}\left(u_{h}^{\theta}\right) \in H_{T, c}^{*}\left(\tau_{T} M^{h} ; \boldsymbol{Q}\right) \cong$ $H_{c}^{*}\left(T \backslash \tau_{T} M^{h} ; Q\right)$ (the subscript $c$ denotes the cohomology with compact support). We define $\operatorname{ch}_{T}^{h}(u) \in H_{T^{\prime}, c}^{*}\left(\tau_{T} M^{h} ; C\right) \cong H_{c}^{*}\left(T \backslash \tau_{T} M^{h} ; C\right)$ by:

$$
\operatorname{ch}_{T}^{h}(u)=\sum_{0 \leqq \theta<2 \pi} e^{i \theta} \operatorname{ch}_{T}\left(u_{h}^{\theta}\right) .
$$

*) In Atiyah [1], this theorem is proved without any restriction on isotropy subgroups.

**) The definition of the multiplicity $m(h)$ in Atiyah [1] is incorrect. It depends on the whole group $T$ and the connected component $M_{i}^{h}$ in $M^{h}$. 
Let $\left.\tau_{T} M\right|_{M^{h}}=\tau_{T} M^{h} \oplus \nu_{h, T}=\tau_{T} M^{h} \oplus\left(\oplus_{0<\theta \leqq \pi} \nu_{h, T}^{\theta}\right)$ be the eigenspace decomposition. $\nu_{n, T}^{\pi}$ is the real eigenvector bundle of eigenvalue -1 and $\nu_{h, T}^{\theta}$ $(0<\theta<\pi)$ is a complex vector bundle on which the action of $h$ is the multiplication by the scalar $e^{i \theta}$. We denote formally the equivariant Pontrjagin classes of $\tau_{T} M^{h}$ and $\nu_{h, T}^{\pi}$ by $p_{T}\left(\tau_{T} M^{h}\right)=\prod\left(1+x_{j}^{2}\right) \in H_{T}^{*}\left(M^{h} ; \boldsymbol{Q}\right)$ and $p_{T}\left(\nu_{n, T}^{\pi}\right)=\Pi\left(1+y_{j}^{2}\right) \in H_{T}^{*}\left(M^{n} ; \boldsymbol{Q}\right)$ respectively, and the equivariant Chern classes of $\nu_{h, T}^{\theta}$ by $c_{T}\left(\nu_{h, T}^{\theta}\right)=\prod\left(1+z_{j}\right) \in H_{T}^{*}\left(M^{h} ; \boldsymbol{Q}\right)$. Then we define $\mathscr{I}_{T}^{h}(M) \in H_{T}^{*}\left(M^{n} ; C\right) \cong H^{*}\left(T \backslash M^{n} ; C\right)$ by:

$$
\mathscr{I}_{T}^{h}(M)=\operatorname{det}_{R}\left(1-\left.h\right|_{\nu_{h, T}}\right)^{-1} \mathscr{R}_{T}\left(\nu_{h, T}^{\pi}\right)\left\{\prod_{0<\theta<\pi} \mathscr{S}_{T}^{\theta}\left(\nu_{h, T}^{\theta}\right)\right\} \mathscr{I}_{T}\left(M^{h}\right),
$$

where

$$
\begin{aligned}
& \mathscr{I}_{T}\left(M^{h}\right)=\mathscr{T}_{T}\left(\tau_{T} M^{h} \otimes_{R} C\right)=\prod_{j}\left(\frac{x_{j}}{1-e^{-x_{j}}} \frac{-x_{j}}{1-e^{x_{j}}}\right), \\
& \mathscr{R}_{T}\left(\nu_{h, T}^{\pi}\right)=\prod_{j}\left(\frac{2}{1+e^{y_{j}}} \frac{2}{1+e^{-y_{j}}}\right), \\
& \mathscr{S}_{T}^{\theta}\left(\nu_{h, T}^{\theta}\right)=\prod_{j}\left(\frac{1-e^{i \theta}}{1-e^{z_{j}+i \theta}} \frac{1-e^{-i \theta}}{1-e^{-z_{j}-i \theta}}\right) .
\end{aligned}
$$

Consider the orbit space $X=T \backslash M$ as a $V$-manifold. By definition, we can see:

$$
\begin{aligned}
& X \amalg\left(\coprod_{i} \Sigma_{i}\right)=T \backslash M \amalg\left(\underset{\substack{h \in T^{h}, M^{h \neq \neq} \\
M_{i}^{h} \subset M^{h}}}{\amalg} T \backslash M_{i}^{h}\right), \\
& \tau_{V} X \amalg\left(\coprod_{i} \tau_{V} \Sigma_{i}\right)=T \backslash \tau_{T} M \amalg\left(\coprod_{h, i} T \backslash \tau_{T} M_{i}^{h}\right) .
\end{aligned}
$$

So we may identify $H_{T, c}^{*}\left(\tau_{T} M ; \boldsymbol{Q}\right)$ with $H_{c}^{*}\left(\tau_{V} X ; \boldsymbol{Q}\right)$ and $H_{T, c}^{*}\left(\tau_{T} M_{i}^{h} ; C\right)$ with $H_{c}^{*}\left(\tau_{V} \Sigma_{i} ; C\right)$. Then, for $u \in K_{V}\left(\tau_{V} X\right) \cong K_{T}\left(\tau_{T} M\right)$, we can interpret:

$$
\operatorname{ch}(u) \mathscr{I}(X)+\operatorname{ch}^{\Sigma}(u) \mathscr{I}^{\Sigma}(X)=\operatorname{ch}_{T}(u) \mathscr{I}_{T}(M)+\sum_{h} \operatorname{ch}_{T}^{h}(u) \mathscr{I}_{T}^{h}(M) .
$$

Thus we have shown that the Atiyah's formula (11) is equivalent to our formula (7), if the $V$-manifold $X$ is obtained as the orbit space of a toral action.

Now we consider a general $V$-manifold $X$. We may assume that $X$ is the orbit space of a $G$-manifold $M . \quad G$ acts on $M$ with only finite isotropy subgroups and of trivial principal orbit type. Then, for a real or complex $G$-equivariant vector bundle $E$, we may identify the $G$-equivariant characteristic class of $E$ with the characteristic class of the $V$-bundle $G \backslash E \rightarrow X$ 
(defined by the same polynomial in Pontrjagin classes or Chern classes). We shall rewrite the formula (7) in the word of equivariant characteristic classes.

By the compactness of $M$ and the smoothness of the $G$-action, the number of orbit types of $G$-manifold $M$ is finite. Also, all the isotropy subgroups are finite, so the number of conjugacy classes of elements of $G$ with non-empty fixed point set is finite. Let $(1),\left(h_{1}\right), \cdots,\left(h_{\rho}\right)$ be such conjugacy classes. Each fixed point set $M^{h}$ admits the action of the centralizer $Z_{G}(h)$. Then the action of $h$ on $\left.\tau M\right|_{M^{h}}$ defines the decomposition into eigenvector bundles

$$
\left.\tau M\right|_{M^{h}}=\tau_{Z_{G(}(h)} M^{h} \oplus \nu_{h, G}=\tau_{Z_{G(h)}} M^{h} \oplus\left(\underset{0<\theta \leqq \pi}{\oplus} \nu_{h, G}^{\theta}\right)
$$

Since $Z_{G}(h)$ commutes with $h$, each summand is $Z_{G}(h)$-equivariant. Then we define $\mathscr{I}_{G}^{h}(M) \in H_{Z_{G}(h)}^{*}\left(M^{h} ; C\right)$ by;

$$
\mathscr{I}_{G}^{h}(M)=\operatorname{det}_{R}\left(1-\left.h\right|_{\nu_{h, G}}\right)^{-1} \mathscr{R}_{Z_{G(h)}}\left(\nu_{h, G}^{\pi}\right)\left\{\prod_{0<\theta<\pi} \mathscr{S}_{Z_{G}(h)}^{\theta}\left(\nu_{h, G}^{\theta}\right)\right\} \mathscr{I}_{Z_{G(h)}\left(M^{h}\right) .}
$$

We remark that $\nu_{h, G}$ and the normal bundle of $M^{n}$ in $M$ differ in dimension equal to $\operatorname{dim} G-\operatorname{dim} Z_{G}(h)$. For $u \in K_{V}\left(\tau_{V} X\right) \cong K_{G}\left(\tau_{G} M\right)$, we have $i_{h}^{*} u \in K_{Z_{G(h)}}\left(\tau_{Z_{G(h)}} M^{h}\right)$ and the eigenspace decomposition $i_{h}^{*} u=\bigoplus_{0 \leqq \theta<2 \pi} u_{h}^{\theta}$. Then we define:

$$
\operatorname{ch}_{G}^{h}(u)=\sum_{0 \leqq \theta<2 \pi} e^{i \theta} \operatorname{ch}_{Z_{G(h)}}\left(u_{h}^{\theta}\right) \in H_{Z_{G(h), c}^{*}}^{*}\left(\tau_{Z_{G}(h)} M^{h} ; C\right) .
$$

Let $\Sigma X=\amalg \Sigma_{i}$ be the singularity $V$-manifold. Then we have canonical identifications

$$
\amalg \Sigma_{i}=\prod_{j=1}^{\rho} Z_{G}\left(h_{j}\right) \backslash M^{h_{j}}, \quad \amalg \tau_{V} \Sigma_{i}=\prod_{j=1}^{\rho} Z_{G}\left(h_{j}\right) \backslash \tau_{Z_{G\left(h_{j}\right)}} M^{h_{j}} .
$$

Let $Z_{G}(h) \backslash M^{h}=\amalg Z_{G}(h) \backslash M_{i}^{h}$ be the decomposition into connected components. Each $M_{i}^{h}$ is $Z_{G}(h)$-invariant but not connected in general. We define the multiplicity $m_{G}\left(M_{i}^{h}\right)$ by:

$$
m_{G}\left(M_{i}^{h}\right)=\text { the order of }\left\{g \in Z_{G}(h) \mid g x=x \text {, for any } x \in M_{i}^{h}\right\} .
$$

Now we can rewrite the formula (7) into:

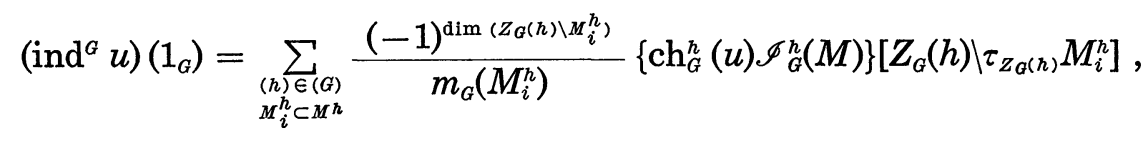


where we denote by $(G)$ the set of conjugacy classes of $G$. We shall deduce this formula from (11) and a computation in the equivariant Chern classes on the flag manifold $G / T$.

\section{§2. Gysin homomorphisms (integrations over the fibre)}

Let $G$ be a compact connected Lie group and let $M$ be a compact $G$-manifold without boundary. We assume that $G$ acts on $M$ with only finite isotropy subgroups. Let $T$ be a maximal torus of $G$. We choose and fix a $G$-invariant complex structure on the flag manifold $G / T$. Then, by (10) and (11), we have, for $u \in K_{G}\left(\tau_{G} M\right)$ :

$$
\begin{aligned}
\left(\operatorname{ind}^{G} u\right)\left(1_{G}\right) & =\left(\operatorname{ind}^{T} r u\right)\left(1_{T}\right) \\
& =\sum_{\substack{h \in T \\
M_{i}^{h} \subset M^{h}}} \frac{(-1)^{\mathrm{dIm}\left(T \backslash \mu_{i}^{h}\right)}}{m_{T}\left(M_{i}^{h}\right)}\left\{\operatorname{ch}_{T}^{h}(r u) \mathscr{I}_{T}^{h}(M)\right\}\left[T \backslash \tau_{T} M_{i}^{h}\right] .
\end{aligned}
$$

Here $M_{i}^{h} \subset M^{h}$ denotes a connected component. In the sequel we omit $i$ 's since all the arguments are parallel.

To deduce (12), we need to reform (13) into the evaluation over $\left[Z_{G}(h) \backslash \tau_{Z_{\theta}(h)} M^{n}\right]^{\prime}$ s. We use the Gysin homomorphisms. Consider the commutative diagram

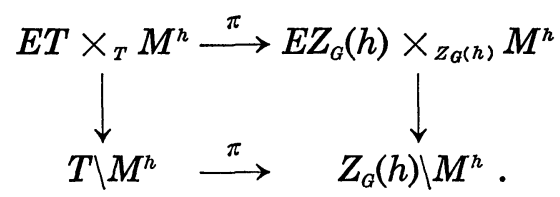

The vertical maps induce the identifications $H^{*}\left(T \backslash M^{n} ; Q\right) \cong H_{T}^{*}\left(M^{n} ; Q\right)$ and $H^{*}\left(Z_{G}(h) \backslash M^{n} ; \boldsymbol{Q}\right) \cong H_{Z_{(j(h)}^{*}}^{*}\left(M^{n} ; \boldsymbol{Q}\right)$. The upper $\pi$ is a fibration with fibre $Z_{\theta}(X) / T$. We orient $Z_{\theta}(h) / T$ by the induced complex structure from $G / T$. We denote the orientation sheaf on $M^{h}$ by $o\left(M^{n}\right)$. Then we have the Gysin homomorphism $\pi_{1}: H_{T}^{*}\left(M^{n} ; o\left(M^{n}\right) \otimes \boldsymbol{Q}\right) \rightarrow H_{Z_{G}(h)}^{*}\left(M^{n} ; o\left(M^{n}\right) \otimes Q\right)$. We may reconstruct $\pi_{1}$ by using the Leray-Serre spectral sequence of the map $\pi: T \backslash M^{h}-Z_{K}(h) \backslash M$. Then we have the following proposition:

Proposition. The Gysin homomorphism $\pi_{1}$ :

$$
H_{T}^{*}\left(M^{h} ; o\left(M^{h}\right) \otimes Q\right) \longrightarrow H_{Z_{G(h)}^{*}}^{*}\left(M^{n} ; o\left(M^{h}\right) \otimes \boldsymbol{Q}\right)
$$

is a $H_{Z_{(h(h)}}^{*}\left(M^{h} ; \boldsymbol{Q}\right)$-module homomorphism. For $x \in H_{T}^{*}\left(M^{h} ; o\left(M^{h}\right) \otimes Q\right)$, we have the following formula: 


$$
\frac{1}{m_{T}\left(M^{n}\right)}\left\langle x,\left[T \backslash M^{n}\right]\right\rangle=\frac{1}{m_{G}\left(M^{n}\right)}\left\langle\pi_{1} x,\left[Z_{G}(h) \backslash M^{h}\right]\right\rangle .
$$

Also we have the Thom isomorphisms

$$
\psi_{T}: H_{T}^{*}\left(M^{h} ; o\left(M^{h}\right) \otimes \boldsymbol{Q}\right) \longrightarrow H_{T, c}^{*}\left(\tau_{T} M^{n} ; \boldsymbol{Q}\right)
$$

and

$$
\psi_{Z_{G(h)}}: H_{Z_{\alpha(h)}}^{*}\left(M^{h} ; o\left(M^{h}\right) \otimes Q\right) \longrightarrow H_{Z_{\alpha(h), c}}^{*}\left(\tau_{Z_{G(h)}} M^{h} ; \boldsymbol{Q}\right) .
$$

Then we define $\tau \pi_{1}=\psi_{Z_{G(h)}} \circ \pi_{1} \circ\left(\psi_{T}\right)^{-1}: H_{T, c}^{*}\left(\tau_{T} M^{h} ; \boldsymbol{Q}\right) \rightarrow H_{Z_{G(h), c}^{*}}^{*}\left(\tau_{Z_{\sigma(h)}} M^{h} ; \boldsymbol{Q}\right)$. It is also a $H_{Z_{\alpha(h)}}^{*}\left(M^{h} ; Q\right)$-homomorphism. Looking carefully at the orientations of $T \backslash \tau_{T} M^{h}$ and $Z_{G}(h) \backslash \tau_{Z_{g(h)}} M^{h}$, we have, for $y \in H_{T, c}^{*}\left(\tau_{T} M^{h} ; Q\right)$ :

$$
\begin{aligned}
\frac{1}{m_{T}\left(M^{h}\right)}\left\langle y,\left[T \backslash \tau_{T} M^{h}\right]\right\rangle & =\frac{(-1)^{m_{h}}}{m_{T}\left(M^{h}\right)}\left\langle\tau \pi_{1} y,\left[Z_{\theta}(h) \backslash \tau_{Z_{G(h)}} M^{n}\right]\right\rangle, \\
\left(m_{h}=\frac{1}{2} \operatorname{dim}_{R}\left(Z_{\theta}(h) / T\right)\right. & \left.=\operatorname{dim}_{C}\left(Z_{\theta}(h) / T\right)\right) .
\end{aligned}
$$

We apply $\tau \pi_{1}$ to $\left\{\operatorname{ch}_{T}^{h}(r u) \mathscr{I}_{T}^{h}(M)\right\}$ in (13). Then we get:

$$
\begin{aligned}
& \left(\operatorname{ind}^{G} u\right)\left(1_{G}\right)=\sum_{h \in T} \frac{\varepsilon_{G}\left(M^{h}\right)}{m_{G}\left(M^{h}\right)}(-1)^{m_{h}} \tau \pi_{1}\left\{\operatorname{ch}_{T}^{h}(r u) \mathscr{I}_{T}^{h}(M)\right\}\left[Z_{G}(h) / \tau_{Z_{\alpha(h)}} M^{h}\right], \\
& \left(\varepsilon_{G}\left(M^{n}\right)=(-1)^{\mathrm{dim}\left(Z_{\left.G(h) M M^{h}\right)}\right) .}\right.
\end{aligned}
$$

We compute each term $(-1)^{m_{h}} \tau \pi_{1}\left\{\operatorname{ch}_{T}^{h}(r u) \mathscr{I}_{T}^{h}(M)\right\}$ independently. First we consider $\mathscr{I}_{T}^{h}(M) \in H_{T}^{*}\left(M^{h} ; C\right)$. We have isomorphisms:

$$
H_{T}^{*}\left(M^{h} ; C\right) \cong H_{Z_{G(h)}^{*}}^{*}\left(Z_{G}(h) \times_{T} M^{h} ; C\right) \cong H_{Z_{G(h)}}^{*}\left(Z_{G}(h) / T \times M^{h}\right) .
$$

Recall the definition:

$$
\mathscr{I}_{T}^{h}(M)=\operatorname{det}_{R}\left(1-\left.h\right|_{\nu_{h}}\right)^{-1} \mathscr{R}_{T}\left(\nu_{h, T}^{\pi}\right)\left\{\prod_{0<\theta<\pi} \mathscr{S}_{T}^{\theta}\left(\nu_{h, T}^{\theta}\right)\right\} \mathscr{I}_{T}\left(M^{h}\right) .
$$

$\nu_{h, T}$ is a $Z_{G}(h)$-equivariant bundle and decomposes equivariantly into:

$$
\nu_{h, T}=\nu_{h, G} \oplus \tau_{0}\left(G / Z_{G}(h)\right),
$$

where $\tau_{0}\left(G / Z_{\theta}(h)\right)$ denotes the tangent space of $G / Z_{\theta}(h)$ at the identity coset. (We denote by the same symbol the vector space and the trivial vector bundle). So, if we lift the $T$-equivariant bundle $\nu_{h, T}$ to a $Z_{G}(h)$ equivariant bundle over $Z_{G}(h) \times_{T} M^{h} \cong Z_{G}(h) / T \times M^{h}$, we may consider it as the pull-back of a $Z_{G}(h)$-equivariant bundle $\nu_{h, T}$ over $M^{h}$. Since $Z_{G}(h) / T$ is a complex submanifold of $G / T, \tau_{0}\left(G / Z_{\theta}(h)\right)$ is a complex vector space with a linear action of $h . h$ does not have any non-zero fixed 
vector on $\tau_{0}\left(G / Z_{G}(h)\right)$. Let $\bigoplus_{0<\theta<2 \pi} \tau_{0}^{\theta}\left(G / Z_{G}(h)\right)$ be the eigenspace decomposition. We define:

$$
\begin{aligned}
\mathscr{I}_{G}^{h}\left(G / Z_{G}(h)\right)_{0}= & \operatorname{det}_{R}\left(1-\left.h\right|_{\tau_{0}\left(G / Z_{G}(h)\right)}\right)^{-1}\left\{\prod_{0<\theta<2 \pi} \mathscr{S}_{Z_{G(h)}^{\theta}}^{\theta}\left(\tau_{0}^{\theta}\left(G / Z_{G}(h)\right)\right)\right\} \\
& \in H_{Z_{G}(h)}^{* *}(p t ; C) .
\end{aligned}
$$

Then we have:

$$
\begin{aligned}
\operatorname{det}_{R} & \left(1-\left.h\right|_{\nu_{h, T}}\right)^{-1} \mathscr{R}_{T}\left(\nu_{h, T}^{\pi}\right)\left\{\prod_{0<\theta<\pi} \mathscr{S}_{T}^{\theta}\left(\nu_{h, T}^{\theta}\right)\right\} \\
& =\mathscr{I}_{G}^{n}\left(G / Z_{G}(h)\right)_{0} \times \operatorname{det}\left(1-\left.h\right|_{\nu_{h, G}}\right)^{-1} \mathscr{R}_{Z_{G}(h)}\left(\nu_{h, G}^{\pi}\right)\left\{\prod_{0<\theta<\pi} \mathscr{S}_{Z_{G}(h)}^{\theta}\left(\nu_{h, G}^{\theta}\right)\right\},
\end{aligned}
$$

where the first factor is in $H_{Z_{G}(h)}^{* *}(p t ; C)$ and the second factor is in $H_{Z_{G}(h)}^{*}\left(M^{h} ; C\right)$. Also we have a $T$-equivariant decomposition:

$$
\tau_{T} M^{h}=\tau_{Z_{G(h)}} M^{h} \oplus \tau_{0}\left(Z_{G}(h) / T\right) .
$$

If we lift $\tau_{T} M^{h}$ over $Z_{G}(h) / T \times M^{h}$, then $\tau_{Z_{G(h)}} M^{h}$ is a $Z_{G}(h)$-equivariant bundle over $M^{h}$ and $\tau_{0}\left(Z_{G}(h) / T\right)$ is the tangent bundle of $Z_{G}(h) / T$. Hence we have:

$$
\mathscr{I}_{T}\left(M^{h}\right)=\mathscr{I}_{Z_{G}(h)}\left(Z_{G}(h) / T\right) \times \mathscr{I}_{Z_{G(h)}}\left(M^{h}\right) .
$$

As a whole, we have:

$$
\begin{gathered}
\mathscr{I}_{T}^{h}(M)=\mathscr{I}_{Z_{G(h)}}\left(Z_{G}(h) / T\right) \times \mathscr{I}_{G}^{h}\left(G / Z_{G}(h)\right)_{0} \times \mathscr{I}_{G}^{h}(M) \\
\in H_{Z_{G(h)}^{*}}^{*}\left(Z_{C}(h) / T \times M^{h} ; C\right),
\end{gathered}
$$

where the first factor is in $H_{Z_{G}(h)}^{* *}\left(Z_{G}(h) / T ; Q\right)$, the second factor is in $H_{Z_{G}(h)}^{* *}(p t ; C)$ and the third factor is in $H_{Z_{G(h)}^{*}}^{*}\left(M^{h} ; C\right)$.

Next we compute $\operatorname{ch}_{T}^{h}(r u) \in H_{T, c}^{*}\left(\tau_{T} M^{h} ; C\right)$. By definition, we have $r u$ $[\bar{\partial}] \times u \in K_{G}\left(\tau(G / T) \times \tau_{G} M\right) \cong K_{T}\left(\tau_{T} M\right)$. So

$$
i_{h}^{*} r u=\left[\left.\bar{\partial}_{G / T}\right|_{Z_{G(h) / T}}\right] \times i_{h}^{*} u \in K_{Z_{G}(h)}\left(\tau\left(Z_{G}(h) / T\right) \times \tau_{Z_{G}(h)} M^{h}\right) .
$$

Since $Z_{G}(h) / T$ is a complex submanifold of $G / T$, we have $\left[\left.\bar{\partial}_{G / T}\right|_{Z_{G}(h) / T}\right]=$ $\left[\bar{\partial}_{Z_{G(h) / T}}\right] \lambda_{-1}\left(\tau_{0}\left(G / Z_{G}(h)\right)\right) \in K_{Z_{G}(h)}\left(\tau\left(Z_{G}(h) / T\right)\right)$. Hence we have:

$$
i_{h}^{*} r u=\left[\bar{\partial}_{Z_{G(h) / T}}\right] \times \lambda_{-1}\left(\tau_{0}\left(G / Z_{G}(h)\right)\right) \times i_{h}^{*} u,
$$

where the first factor is in $K_{Z_{G}(h)}\left(\tau\left(Z_{G}(h) / T\right)\right)$, the second factor is in $R\left(Z_{G}(h)\right)$ and the third factor is in $K_{Z_{G}(h)}\left(\tau_{Z_{G}(h)} M^{n}\right)$. Consider the eigenspace decomposition by the action of $h$. The action is trivial on $Z_{G}(h) / T$. So we have: 


$$
\sum e^{i \theta}\left(i_{h}^{*} r u\right)^{\theta}=\left[\bar{\partial}_{Z_{G(h) / T}}\right] \times\left(\sum e^{i \theta} \lambda_{-1}\left(\tau_{0}^{\theta}\left(G / Z_{G}(h)\right)\right)\right) \times\left(\sum e^{i \theta}\left(i_{h}^{*} u\right)^{\theta}\right) .
$$

Applying the Chern character on both sides, we have:

$$
\begin{aligned}
\operatorname{ch}_{T}^{h}(r u)= & \operatorname{ch}_{Z_{G(h)}}\left[\bar{\partial}_{Z_{G(h) / T}}\right] \times \operatorname{ch}_{G}^{h}\left(\lambda_{-1}\left(\tau_{0}\left(G / Z_{G}(h)\right)\right)\right) \times \operatorname{ch}_{G}^{h}(u) \\
& \in H_{T, c}^{*}\left(\tau_{T} M^{h} ; C\right) \cong H_{Z_{G}(h), c}^{*}\left(\tau\left(Z_{G}(h) / T\right) \times \tau_{Z_{\sigma(h)}} M^{h} ; C\right),
\end{aligned}
$$

where the first factor is in $H_{Z_{G}(h), c}^{* *}\left(\tau\left(Z_{G}(h) / T\right) ; C\right)$, the second factor is in $H_{Z_{G(h)}^{*}}^{* *}(p t ; C)$ and the third factor is in $H_{Z_{g(h), c}^{*}}^{*}\left(\tau_{Z_{\sigma(h)}} M^{n} ; C\right)$. Combining this with the computation on $\mathscr{I}_{T}^{h}(M)$, we have:

$$
\begin{aligned}
\operatorname{ch}_{T}^{h}(r u) \mathscr{I}_{T}^{h}(M)= & \operatorname{ch}_{Z_{G(h)}}\left[\bar{\partial}_{Z_{(h)}(h) / T}\right] \mathscr{I}_{Z_{G(h)}}\left(Z_{G}(h) / T\right) \\
& \times \operatorname{ch}_{G}^{h}\left(\lambda_{-1}\left(\tau_{0}\left(G / Z_{G}(h)\right)\right)\right) \mathscr{I}_{G}^{h}\left(G / Z_{G}(h)\right)_{0} \\
& \times \operatorname{ch}_{G}^{h}(u) \mathscr{I}_{G}^{h}(M) \\
& \in H_{T, c}^{*}\left(\tau_{T} M^{h} ; C\right) \cong H_{Z_{G(h), c}}^{*}\left(\tau\left(Z_{G}(h) / T\right) \times \tau_{\left.Z_{G(h)}\right)} M^{h} ; C\right),
\end{aligned}
$$

where the first factor is in $H_{Z_{G}(h), c}^{*}\left(\tau\left(Z_{G}(h) / T ; Q\right)\right.$, the second factor is in $H_{Z_{G}(h)}^{* *}(p t ; C)$ and the third factor is in $H_{Z_{G(h), c}}^{*}\left(\tau_{Z_{G}(h)} M^{h} ; C\right)$. We have also:

$\operatorname{ch}_{Z_{G(h)}}\left[\bar{\partial}_{Z_{G(h) / T}}\right] \mathscr{I}_{Z_{G(h)}}\left(Z_{G}(h) / T\right)=(-1)^{m_{h}} \psi\left(\mathscr{T}_{Z_{\sigma}(h)}\left(Z_{G}(h) / T\right)\right)$,

$\left(\psi: H_{Z_{G}(h)}^{* *}\left(Z_{G}(h) / T ; Q\right) \longrightarrow H_{Z_{G}(h), c}^{* *}\left(\tau\left(Z_{G}(h) / T\right) ; Q\right)\right.$, Thom isomorphism),

$\operatorname{ch}_{G}^{h}\left(\lambda_{-1}\left(\tau_{0}\left(G / Z_{G}(h)\right)\right)\right) \mathscr{I}_{G}^{h}\left(G / Z_{G}(h)\right)_{0}=\mathscr{T}_{G}^{h}\left(G / Z_{G}(h)\right)_{0}$,

(the residual Todd class restricted at the identity component).

By the identification $H_{T, c}^{*}\left(\tau_{T} M^{n} ; C\right) \cong H_{Z_{g(h), c}^{*}}^{*}\left(\tau\left(Z_{G}(h) / T\right) \times \tau_{Z_{G(h)}} M^{h} ; C\right), \tau \pi_{1}$ is given by the composite:

$$
\begin{aligned}
\tau \pi_{1}: & H_{Z_{G(h), c}^{*}}^{*}\left(\tau\left(Z_{G}(h) / T\right) \times \tau_{Z_{G(h)}} M^{h} ; C\right) \\
& \stackrel{\psi^{-1}}{\longrightarrow} H_{Z_{G(h)}}^{*}\left(Z_{G}(h) / T \times M^{h} ; o\left(M^{h}\right) \otimes C\right) \\
& \stackrel{\pi_{1}}{\longrightarrow} H_{Z_{G(h)}}^{*}\left(M^{h} ; o\left(M^{h}\right) \otimes C\right) \\
& \stackrel{\psi}{\longrightarrow} H_{Z_{G(h), c}}^{*}\left(\tau_{Z_{G(h)}} M^{h} ; C\right) .
\end{aligned}
$$

Then we can see:

$$
\begin{aligned}
& (-1)^{m_{h}} \tau \pi_{!}\left\{\operatorname{ch}_{T}^{h}(r u) \mathscr{I}_{T}^{h}(M)\right\} \\
& \quad=\pi_{1}\left\{\mathscr{T}_{Z_{G(h)}(h)}\left(Z_{G}(h) / T\right) \mathscr{T}_{G}^{h}\left(G / Z_{G}(h)\right)_{0}\right\} \operatorname{ch}_{G}^{h}(u) \mathscr{I}_{G}^{h}(M), \\
& \left(\pi_{!}: H_{Z_{G}(h)}^{* *}\left(Z_{G}(h) / T ; C\right) \longrightarrow H_{Z_{G}(h)}^{*}(p t ; C)\right) .
\end{aligned}
$$

Thus we have proved:

$$
\begin{aligned}
\left(\operatorname{ind}^{G} u\right)\left(1_{G}\right)=\sum_{\substack{h \in T \\
M \neq \neq}} \frac{\varepsilon_{G}\left(M^{h}\right)}{m_{G}\left(M^{h}\right)}\left\{\pi_{1}\left\{\mathscr{T}_{Z_{G}(h)}\left(Z_{G}(h) / T\right) \mathscr{T}_{G}^{h}\left(G / Z_{G}(h)\right)_{0}\right\}\right. \\
\left.\quad \times \operatorname{ch}_{G}^{h}(u) \mathscr{I}_{G}^{h}(M)\right\}\left[Z_{G}(h) \backslash \tau_{Z_{G(h)}} M^{h}\right] .
\end{aligned}
$$


We compare this formula with the final form (12). In (12), the summation moves over the conjugacy classes $(h)$ in $G$ such that $M^{h} \neq \emptyset$, but in (15), the summation moves over all the elements in $T$ such that $M^{h}$ $\neq \emptyset$. We recall that every conjugacy class $(h)$ in $G$ meets $T$ by finite (non-zero) times. So in (15), we sum up first the terms corresponding to the elements that belong to the same conjugacy class in $G$.

Let $h$ and $h^{\prime}$ be elements in $T$ conjugate in $G$. Choose $g \in G$ such that $g h g^{-1}=h^{\prime}$. We denote by $\phi_{g}$ the action of $g$ on $M$ and by $\iota_{g}$ the inner automorphism induced by $g$. Then $\phi_{g}: M \rightarrow M$ is $\iota_{g}$-equivariant and maps $M^{h}$ onto $M^{h^{\prime}}$. It induces bundle equivalences $\tau_{h} \phi_{g}: \tau_{Z_{G(h)}} M^{h} \rightarrow$ $\tau_{Z_{G\left(h^{\prime}\right)}} M^{h^{\prime}}$ and $\nu_{h} \phi_{g}: \nu_{h, G} \rightarrow \nu_{h^{\prime}, G}$. These equivalences are $\left[\iota_{g}: Z_{G}(h) \rightarrow Z_{G}\left(h^{\prime}\right)\right]-$ equivariant. This shows $\phi_{g}^{*} \mathscr{I}_{G}^{h^{\prime}}(M)=\mathscr{I}_{G}^{h}(M)$ and $\left(\tau_{h} \phi_{g}\right)^{*} \operatorname{ch}_{G}^{h^{\prime}}(u)=\operatorname{ch}_{G}^{h}(u)$. Hence we have:

$$
\begin{aligned}
\left(\tau_{h} \phi_{g}\right) & *\left\{\pi_{1}\left\{\mathscr{T}_{Z_{G\left(h^{\prime}\right)}}\left(Z_{G}\left(h^{\prime}\right) / T\right) \mathscr{T}_{G}^{h^{\prime}}\left(G / Z_{G}\left(h^{\prime}\right)\right)_{0}\right\} \operatorname{ch}_{G}^{h^{\prime}}(u) \mathscr{I}_{G}^{h^{\prime}}(M)\right\} \\
& =\left\{\iota_{g}^{*} \pi_{1}\left\{\mathscr{T}_{Z_{G\left(h^{\prime}\right)}}\left(Z_{G}\left(h^{\prime}\right) / T\right) \mathscr{T}_{G}^{h^{\prime}}\left(G / Z_{G}\left(h^{\prime}\right)\right)_{0}\right\}\right\} \operatorname{ch}_{G}^{h}(u) \mathscr{I}_{G}^{h}(M) .
\end{aligned}
$$

For each conjugacy class $(h)$ in $G$, we put $(h) \cap T=\left\{h_{1}, h_{2}, \cdots, h_{w(h)}\right\}$. For each $j$, we choose $g_{j} \in G$ such that $h_{j}=g_{j} h g_{j}^{-1}$. Then we have:

$$
\begin{aligned}
\text { (ind } \left.^{G} u\right)\left(1_{G}\right)= & \sum_{\substack{(h) \in G(G) \\
M^{h \neq \emptyset}}} \frac{\varepsilon_{G}\left(M^{h}\right)}{m_{G}\left(M^{h}\right)}\left\{\left\{\sum _ { j = 1 } ^ { w ( h ) } \iota _ { g j } ^ { * } \pi _ { 1 } \left\{\mathscr{T}_{Z_{G(}\left(h_{j}\right)}\left(Z_{G}\left(h_{j}\right) / T\right)\right.\right.\right. \\
& \left.\left.\left.\times \mathscr{T}_{G}^{h_{j}}\left(G / Z_{G}\left(h_{j}\right)\right)_{0}\right\}\right\} \operatorname{ch}_{G}^{h}(u) \mathscr{I}_{G}^{h}(M)\right\}\left[Z_{G}(h) \backslash \tau_{Z_{G(h)}} M^{h}\right] .
\end{aligned}
$$

Now we consider the class

$$
\sum_{j=1}^{w(h)} \iota_{g_{j}}^{*} \pi_{!}\left\{\mathscr{T}_{Z_{G\left(h_{j}\right)}}\left(Z_{G}\left(h_{j}\right) / T\right) \mathscr{T}_{G}^{h_{j}}\left(G / Z_{G}\left(h_{j}\right)\right)_{0}\right\} \in H_{Z_{G}(h)}^{* *}(p t ; C) .
$$

The action of $h$ on $\tau_{0}\left(G / Z_{G}(h)\right)$ has no fixed non-zero vector. By an elementary consideration, we have:

$$
(G / T)^{h}=\prod_{j=1}^{w(h)} g_{j}^{-1} Z_{G}\left(h_{j}\right) / T .
$$

Recall the definition of $\mathscr{T}_{G}^{h}(G / T) \in H_{Z_{G}(h)}^{* *}\left((G / T)^{h} ; C\right)$. We can see that $\mathscr{T}_{Z_{G(h)}}\left(Z_{G}(h) / T\right) \mathscr{T}_{G}^{h}\left(G / Z_{G}(h)\right)_{0}$ is the restriction of $\mathscr{T}_{G}^{h}(G / T)$ onto the component $Z_{G}(h) / T$. The holomorphic action of $g_{j}$ on $G / T$ defines a map $\psi_{g_{j}}: g_{j}^{-1} Z_{G}\left(h_{j}\right) / T \rightarrow Z_{G}\left(h_{j}\right) / T$. It is $\iota_{g_{j}}$-equivariant. Hence we have:

$$
\begin{aligned}
\iota_{g_{j}}^{*} \pi_{1}\left\{\mathscr{T}_{Z_{G\left(h_{j}\right)}}\left(Z_{G}\left(h_{j}\right) / T\right) \mathscr{T}_{G}^{h_{j}}\left(G / Z_{G}\left(h_{j}\right)\right)_{0}\right\} & \\
& =\pi_{1}\left\{\psi_{g_{j}}^{*} \mathscr{T}_{Z_{G\left(h_{j}\right)}}\left(Z_{G}\left(h_{i}\right) / T\right) \mathscr{T}_{G}^{h_{j}}\left(G / Z_{G}\left(h_{j}\right)\right)_{0}\right\} \\
& =\pi_{!}\left(\left.\mathscr{T}_{G}^{h}(G / T)\right|_{g_{j}^{-1} Z_{G}\left(h_{j}\right) / T}\right) .
\end{aligned}
$$


Thus we have proved:

$$
\begin{aligned}
& \left(\text { ind }^{G} u\right)\left(1_{G}\right) \\
& \quad=\sum_{\substack{(h) \in G(G) \\
M}} \frac{\varepsilon_{G}\left(M^{h}\right)}{m_{G}\left(M^{h}\right)}\left\{\pi_{!}\left(\mathscr{T}_{G}^{h}(G / T)\right) \operatorname{ch}_{G}^{h}(u) \mathscr{I}_{G}^{h}(M)\right\}\left[Z_{G}(h) \backslash \tau_{Z_{G}(h)} M^{h}\right], \\
& \left(\pi_{!}: H_{Z_{G(h)}^{*}}^{* *(}\left((G / T)^{h} ; C\right) \longrightarrow H_{Z_{G}(h)}^{* *}(p t ; C)\right) .
\end{aligned}
$$

To complete the proof it will suffice to show:

$$
\pi_{1}\left(\mathscr{T}_{G}^{h}(G / T)\right)=1 \in H_{Z_{G}(h)}^{* *}(p t ; C) .
$$

This will be done in the next section.

\section{§3. Equivariant residual Todd classes over flag manifolds}

Let $G$ be a compact connected Lie group and let $T$ be a maximal torus of $G$. Choose and fix a $G$-invariant complex structure on the flag manifold $G / T$. Let $h \in T$ be an element. Then the fixed point set $(G / T)^{h}$ is a complex submanifold (closed but not connected in general). It admits the holomorphic action of the centralizer $Z_{G}(h)$. Let $E(=E G) \rightarrow E / G(=B G)$ be the universal $G$-principal bundle. Then we have an associated bundle: $E \times_{Z_{G(h)}}(G / T)^{h} \rightarrow E / Z_{G}(h)\left(=B Z_{G}(h)\right)$. Over its total space $E \times_{Z_{G(h)}}(G / T)^{h}$, we have vector bundles

$$
\begin{aligned}
& \tau\left((G / T)^{h}\right)_{z_{G(h)}}=E \times_{z_{G(h)}} \tau\left((G / T)^{h}\right), \\
& \nu^{\theta}\left((G / T)^{h}\right)_{z_{G(h)}}=E \times_{Z_{G(h)}} \nu^{\theta}\left((G / T)^{h}\right) \quad(0<\theta<2 \pi),
\end{aligned}
$$

( $\nu^{\theta}$ denotes the eigenvector bundle by the action of $h$ ). Then we define:

$$
\begin{aligned}
\mathscr{T}_{G}^{h}(G / T)= & \mathscr{T}\left(\tau\left((G / T)^{h}\right)_{\left.z_{G(h)}\right)} \prod_{0<\theta<2 \pi} \mathscr{T}^{\theta}\left(\nu^{\theta}\left((G / T)^{h}\right)_{Z_{G(h)}}\right)\right. \\
& \in H^{* *}\left(E \times_{Z_{G^{(h)}}}(G / T)^{h} ; C\right)=H_{Z_{G(h)}^{*}}^{*}\left((G / T)^{h} ; C\right) .
\end{aligned}
$$

$\pi: E \times_{Z_{G}(h)}(G / T)^{h} \rightarrow E / Z_{G}(h)$ defines the Gysin homomorphism

$$
\pi_{!}: H^{* *}\left(E \times_{Z_{G(h)}}(G / T)^{h} ; C\right) \longrightarrow H^{* *}\left(E / Z_{G}(h) ; C\right) .
$$

The purpose of this section is to prove the following formula

$$
\pi_{!} \mathscr{T}_{G}^{h}(G / T)=1 \in H^{* *}\left(E / Z_{G}(h) ; C\right)=H_{Z}^{* *(h)}(p t ; C) .
$$

This is the last formula in the previous section.

Let $Z_{G}(h)_{0} \subset Z_{G}(h)$ denote the identity component. Then the projection $E / Z_{G}(h)_{0} \rightarrow E / Z_{G}(h)$ is a finite regular covering. The induced map 
$H^{* *}\left(E / Z_{G}(h) ; C\right) \rightarrow H^{* *}\left(E / Z_{G}(h)_{0} ; C\right)$ is injective. So we may reduce the structure group $Z_{G}(h)$ to $Z_{G}(h)_{0}$. We denote by $\pi^{\prime}$ the projection

$$
\pi^{\prime}: E \times_{Z_{G(h)}(h)}(G / T)^{h} \longrightarrow E / Z_{G}(h)_{0} .
$$

Then it will suffice to show:

$$
\pi_{!}^{\prime} \mathscr{T}_{G}^{h}(G / T)=1 \in H^{* *}\left(E / Z_{G}(h)_{0} ; C\right) .
$$

Let $W(G)=N_{G}(T) / T$ and $W\left(Z_{G}(h)_{0}\right)=N_{Z_{q}(h)_{0}}(T) / T$ be the Weyl group of $G$ and $Z_{G}(h)_{0}$ respectively. For each right coset $\left[w_{j}\right]$ in $W(G) / W\left(Z_{G}(h)_{0}\right)$, choose one representative $g_{j} \in N_{G}(T)$. Then, as a $Z_{G}(h)_{0}$-manifold, $(G / T)^{h}$ decomposes into a disjoint union

$$
(G / T)^{n}=\prod_{\left[w_{j}\right] \in W(G) / W\left(Z_{G}(h)_{0}\right)}\left(Z_{G}(h)_{0} g_{j}^{-1}\right) / T .
$$

Put $h_{j}=g_{j} h g_{j}^{-1}$, then the holomorphic action of $g_{j}$ maps $\left(Z_{G}(h)_{0} g_{j}^{-1}\right) / T$ onto $Z_{G}\left(h_{j}\right)_{0} / T$. This map is $\left[c_{g}: Z_{G}(h)_{0} \rightarrow Z_{G}\left(h_{j}\right)_{0}\right]$-equivariant. Over each component $\left(Z_{G}(h)_{0} g_{j}^{-1}\right) / T$ in $(G / T)^{h}$, we may translate everything onto $Z_{G}\left(h_{j}\right)_{0} / T$ by the action of $g_{j}$. Then the bundles

$$
E \times_{Z_{Q(h) 0}} \tau\left((G / T)^{h}\right) \text { and } E \times_{z_{Q(h)} \nu^{o}\left((G / T)^{h}\right)}
$$

are translated to:

$$
\begin{aligned}
& E \times_{Z_{G\left(h_{j}\right)_{0}} \tau} \tau\left(Z_{G}\left(h_{j}\right)_{0} / T\right) \cong E \times_{T} \tau_{0}\left(Z_{G}\left(h_{j}\right)_{0} / T\right),
\end{aligned}
$$

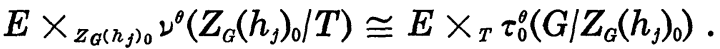

Then we have:

$$
\begin{aligned}
& \pi_{:}^{\prime}\left\{\left.\mathscr{T}_{G}^{h}(G / T)\right|_{\left(Z_{\left.G(h) \delta_{j} j^{1}\right) / T}\right\}}\right. \\
& \quad=\iota_{g_{j}^{\prime}}^{*}\left(\pi_{j}\right)_{1}\left\{\mathscr{T}_{T}\left(\tau_{0}\left(Z_{G}\left(h_{j}\right)_{0} / T\right)\right) \prod_{0<\theta<2 \pi} \mathscr{T}_{T}^{\theta}\left(\tau_{0}^{\theta}\left(G / Z_{G}\left(h_{j}\right)_{0}\right)\right)\right\}, \\
& \left(\pi_{j}: E / T \longrightarrow E / Z_{G}\left(h_{j}\right)_{0}, \iota_{\iota_{j}}: E / Z_{G}(h)_{0} \longrightarrow E / Z_{G}\left(h_{j}\right)_{0}\right) .
\end{aligned}
$$

We can describe these classes in terms of the roots of $G$. Let $a_{1}, a_{2}, \cdots, a_{m}$ be the positive roots of $G$, corresponding to the invariant complex structure on $G / T$ (see Borel-Hirzegruch [4]). Let $g$ be the Lie algebra of $G$ and let $\mathfrak{g}=\mathfrak{h} \oplus \mathfrak{a}_{1} \oplus \mathfrak{a}_{2} \oplus \cdots \oplus \mathfrak{a}_{m}$ be the root space decomposition. That is: $\mathfrak{g}=\tau_{0}(G)$ and $\mathfrak{h}=\tau_{0}(T)$. T acts on $\mathfrak{g}$ by the conjugacy. $\mathfrak{g}=\mathfrak{h} \oplus \mathfrak{a}_{1} \oplus \mathfrak{a}_{2} \oplus \cdots \oplus \mathfrak{a}_{m}$ is the irreducible decomposition of this $T$-action. $\mathfrak{h}$ is the trivial summand. $a_{k}(k=1,2, \cdots, m)$ is a linear functional on $\mathfrak{h}$ such that, on $\mathfrak{a}_{k} \cong C$, the action of $T$ is given by: 


$$
\begin{aligned}
& h z=e^{2 \pi i a_{k}(H)} z, \\
& \left(h \in T, z \in \mathfrak{a}_{k} \cong C, H \in \mathfrak{h} \text { such that } \exp H=h\right) .
\end{aligned}
$$

For the fixed $h \in T$, we choose $H \in \mathfrak{h}$ such that $\exp H=h$ and we put $H_{j}=w_{j} H=\operatorname{Ad}\left(g_{j}\right) H$. Then the $T$-invariant subspaces $\tau_{0}\left(Z_{G}\left(h_{j}\right)_{0} / T\right)$ and $\tau_{0}^{\theta}\left(G / Z_{G}\left(h_{j}\right)_{0}\right)$ in $\tau_{0}(G / T)=\mathfrak{g} / \mathfrak{h}=\mathfrak{a}_{1} \oplus \mathfrak{a}_{2} \oplus \cdots \oplus \mathfrak{a}_{m}$ are given by:

$$
\begin{aligned}
& \tau_{0}\left(Z_{G}\left(h_{j}\right)_{0} / T\right)=\underset{\substack{k ; a_{k}\left(H_{j}\right) \equiv 0 \\
\bmod Z}}{\bigoplus} \mathfrak{a}_{k}, \\
& \tau_{0}^{\theta}\left(G / Z_{G}\left(h_{j}\right)_{0}\right)=\underset{\substack{k ; a_{k}\left(H_{j}\right) \equiv \theta / 2 \pi \\
\bmod \bar{Z}}}{\bigoplus} \mathfrak{a}_{k} .
\end{aligned}
$$

By Borel-Hirzebruch [4], we may identify $H^{* *}(B T ; R)=H^{* *}(E / T ; R)$ with the completion of the symmetric tensor algebra $S^{* *}\left(\mathfrak{h}^{*}\right)$. We denote by $\left[a_{k}\right] \in H^{2}(E / T ; R)$ the corresponding class to $a_{k} \in \mathfrak{h}^{*}$. Then the equivariant total Chern classes are written by:

$$
\begin{aligned}
& c_{T}\left(\tau_{0}\left(Z_{G}\left(h_{j}\right)_{0} / T\right)\right)=\prod_{k ; a_{k}\left(H_{j}\right) \equiv 0}\left(1+\left[a_{k}\right]\right) \in H^{* *}(E / T ; R), \\
& c_{T}\left(\tau_{0}^{\theta}\left(G / Z_{G}\left(h_{j}\right)_{0}\right)\right)=\prod_{k ; a_{k}\left(H_{j}\right) \equiv \theta / 2 \pi}\left(1+\left[a_{k}\right]\right) \in H^{* *}(E / T ; R) .
\end{aligned}
$$

Hence we have:

$$
\begin{aligned}
& \mathscr{T}_{T}\left(\tau_{0}\left(Z_{G}\left(h_{j}\right)_{0} / T\right)\right) \prod_{0<\theta<2 \pi} \mathscr{T}_{T}^{\theta}\left(\tau_{0}^{\theta}\left(G / Z_{G}\left(h_{j}\right)_{0}\right)\right) \\
& \quad=\prod_{k ; a_{k}\left(H_{j}\right) \equiv 0} \frac{\left[a_{k}\right]}{1-e^{-\left[a_{k}\right]}} \prod_{0<\theta<2 \pi k} \prod_{k, a_{k}\left(H_{j}\right) \equiv \theta / 2 \pi} \frac{1}{1-e^{-\left[a_{k}\right]-i \theta}} \\
& \quad=\left\{\prod_{k ; a_{k}\left(H_{j}\right) \equiv 0}\left[a_{k}\right]\right\}\left\{\prod_{k=1}^{m} \frac{1}{1-e^{-\left[a_{k}\right]-2 \pi i a_{k}\left(H_{j}\right)}}\right\}
\end{aligned}
$$

By Borel-Hirzebruch [5], we can compute the Gysin homomorphism $\left(\pi_{j}\right)_{1}$. We remark that $\left\{a_{k} \mid a_{k}\left(H_{j}\right) \equiv 0 \bmod Z\right\}$ are the positive roots of $Z_{G}\left(h_{\jmath}\right)_{0}$. Then we have:

$$
\begin{aligned}
\left\{\prod_{k ; a_{k}\left(H_{j}\right) \equiv 0}\left[a_{k}\right]\right\}\left(\pi_{j}\right)_{1}\left\{\mathscr{T}_{T}\left(\tau_{0}\left(Z_{G}\left(h_{j}\right)_{0} / T\right)\right)\right. & \left.\prod_{0<\theta<2 \pi} \mathscr{T}_{T}^{\theta}\left(\tau_{0}^{\theta}\left(G / Z_{G}\left(h_{j}\right)_{0}\right)\right)\right\} \\
= & \sum_{w \in W\left(Z_{G}\left(h_{j}\right)_{0}\right)} \operatorname{sgn}(w)\left\{\prod_{k ; a_{k}\left(H_{j}\right) \equiv 0}\left[w a_{k}\right]\right\}\left\{\prod_{k=1}^{m} \frac{1}{1-e^{-\left[w a_{k}\right]-2 \pi i a_{k}\left(H_{j}\right)}}\right\} .
\end{aligned}
$$

For $w \in W\left(Z_{G}\left(h_{j}\right)_{0}\right)$, we have:

$$
\begin{aligned}
& \operatorname{sgn}(w)\left\{\prod_{k ; a_{k}\left(H_{j}\right) \equiv 0}\left[w a_{k}\right]\right\}=\prod_{k ; a_{k}\left(H_{j}\right) \equiv 0}\left[a_{k}\right], \\
& w a_{k}\left(H_{j}\right)=a_{k}\left(w^{-1} H_{j}\right)=a_{k}\left(H_{j}\right) \quad(k=1,2, \cdots, m) .
\end{aligned}
$$

Hence we have: 


$$
\begin{gathered}
\left(\pi_{j}\right)_{1}\left\{\mathscr{T}_{T}\left(\tau_{0}\left(Z_{G}\left(h_{j}\right)_{0} / T\right)\right) \prod_{0<\theta<2 \pi} \mathscr{T}_{T}^{\theta}\left(\tau_{0}^{\theta}\left(G / Z_{G}\left(h_{i}\right)_{0}\right)\right)\right\} \\
=\sum_{w \in W\left(Z_{G}\left(h_{j}\right)_{0}\right)} \prod_{k=1}^{m} \frac{1}{1-e^{-\left[w a_{k}\right]-2 \pi i w a_{k}\left(H_{j}\right)}} \cdot
\end{gathered}
$$

The conjugation $\iota_{g_{j}}: E / Z_{G}(h)_{0} \rightarrow E / Z_{G}\left(h_{j}\right)_{0}$ is covered by the map $\iota_{g_{j}}: E / T \rightarrow$ $E / T$. So, in cohomology, $\iota_{g j}^{*}$ is given by the action of the element $w_{j}^{-1} \in$ $W(G)$. Then we have:

$$
\begin{aligned}
\pi_{!}^{\prime} \mathscr{T}_{G}^{h}(G / T) & =\sum_{j} \iota_{g_{j}}^{*}\left(\pi_{j}\right)_{1}\left\{\mathscr{T}_{T}\left(\tau_{0}\left(Z_{G}\left(h_{j}\right)_{0} / T\right)\right) \prod_{\theta} \mathscr{T}_{T}^{\theta}\left(\tau_{0}^{\theta}\left(G / Z_{G}\left(h_{j}\right)_{0}\right)\right)\right\} \\
& =\sum_{\substack{\left[w_{j}\right] \in W(G) / W\left(Z_{G}(h)_{0}\right) \\
w \in W\left(Z_{G}\left(h_{j}\right)_{0}\right)}} \prod_{k=1}^{m} \frac{1}{1-e^{-\left[w_{j}^{-1} w a_{k}\right]-2 \pi i w a_{k}\left(H_{j}\right)}} .
\end{aligned}
$$

Here, $w a_{k}\left(H_{j}\right)=w a_{k}\left(w_{j} H\right)=w_{j}^{-1} w a_{k}(H)$ and in summation $w_{j}^{-1} w$ move just all over $W(G)$. Hence:

$$
\pi_{!}^{\prime} \mathscr{T}_{G}^{h}(G / T)=\sum_{w \in W(G)} \prod_{k=1}^{m} \frac{1}{1-e^{-\left[w a_{k}\right]-2 \pi i w a_{k}(H)}} .
$$

Recall the Weyl's relation that was used in Borel-Hirzebruch [4]. That is, as a function in $X \in \mathfrak{h}$, we have:

$$
\sum_{w \in W(G)} \prod_{k=1}^{m} \frac{1}{1-e^{-w a_{k}(X)}} \equiv 1 .
$$

Replace $X$ by $X+2 \pi i H$ and we get:

$$
\sum_{w \in W(G)} \prod_{k=1}^{m} \frac{1}{1-e^{-w a_{k}(X)-2 \pi i w a_{k}(H)}} \equiv 1 .
$$

The formal power series expansion of this expression gives a relation in $S^{* *}\left(\mathfrak{h}^{*}\right) \otimes C=H^{* *}(E / T ; C)$. This shows:

$$
\pi_{1} \mathscr{T}_{G}^{h}(G / T)=1 \in H^{* *}\left(E / Z_{G}(h) ; C\right) \subset H^{* *}(E / T ; C) .
$$

\section{REFERENCES}

[1] M. F. Atiyah, Elliptic operators and compact groups, Lecture Notes in Math., 401, Springer-Verlag, 1974.

[2] M. F. Atiyah and I. M. Singer, The index of elliptic operators, I, Ann. of Math., 87 (1968), 484-530.

[ 3 ] — The index of elliptic operators, III, Ann. of Math., 87 (1968), 546-604.

[4] A. Borel and F. Hirzebruch, Characteristic classes and homogeneous spaces, I, Amer. J. Math., 80 (1958), 458-538.

[ 5 ] _ C Characteristic classes and homogeneous spaces, II, Amer. J. Math., 81 (1959), 315-382. 
[6] T. Kawasaki, The signature theorem for $V$-manifolds, Topology, 17 (1978), 75-83.

[ 7 ] - The Riemann-Roch theorem for complex $V$-manifolds, Osaka J. Math., 16 (1979), 151-159.

Department of Mathematics

Faculty of Science

Gakushuin University

Mejiro, Tokyo, 171 Japan 